\title{
On Vanishing Coaxial Minors
}

By Тн. Mотzкin, Hebrew University, Jerusalem.

Communicated by H. W. Turnbull.

(Received 26th August, 1935. Read 13th November, 1935.)

I. Introduction.

\$1. In this note we wish to study some properties of a square matrix of order $n$ and of rank $r$,

1.1 .

$$
A=\left(a_{i k}\right), \quad i, k=1,2, \ldots, n,
$$

whose coaxial minors $|M|$ of certain orders, or some of them, are known to be zero. Our main results will lie in two directions, according as the vanishing minors are, within certain limits, of an arbitrary order (Part II) or of two consecutive orders only (Part III). In Part II we will prove the two following theorems:

1.2. If all coaxial minors of an order less than $n-m$ of a square matrix $A$ of order $n>3 m$ vanish, the upper bound ${ }^{1}$ for the number of non-vanishing coaxial minors of order $n-m$ of $A$ is $2^{m}$.

1.3. A square matrix $A$ of order $n>3$ whose coaxial minors of order less than $n-1$ all vanish ${ }^{2}$, can by permuting its rows and columns in the same manner be brought into one of the six following forms:

1.31. $a_{i k}=0$ for $i \geqq k$, other $a_{i k}$ arbitrary;

1.32. $a_{n 1} \neq 0$, all $a_{i+1} \neq 0$, while all other $a_{i k}$ vanish;

1.33. $a_{n 2} \neq 0$, all $a_{i+1} \neq 0$ except $a_{12}$, all $a_{1 k}$ except $a_{11}$ are arbitrary, while all other $a_{i k}$ vanish;

1.34. $=1.33$, reflected at the main diagonal;

1.35. = 1.32, modified by $a_{23}=0, a_{13} \neq 0, a_{24} \neq 0$;

1 This is at most one half and generally a much smaller fraction of the number ${ }_{n} C_{m}$ of all coaxial minors of the same order.

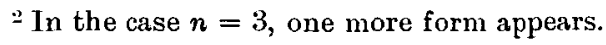


1.36. $=1.35$ with $a_{23} \neq 0$.

For the above cases, there are 0, 0, 1, 1, 2, 2 non-vanishing coaxial first minors of $A$ respectively, whilst $|A|=0$ except for 1.32 and 1.36 .

$\S 2$. If only minors of two consecutive orders of $A$ are known to be zero, we have Kantor's theorem ${ }^{1}$ :

2.1. If all coaxial minors of order $m$ of 1.1 are not zero, while those of orders $m+1$ and $m+2$ all vanish, then $r=m$.

Let us suppose now that $M$ is a given coaxial minor of $A$ and $M_{-}$is a given coaxial first minor of $M$. By the sign + we shall denote simple coaxial bordering; i.e. $M^{+}$symbolises every coaxial minor of $A$ which has $M$ for first minor, so $M$ is a $M_{-}{ }_{+}$. It will be

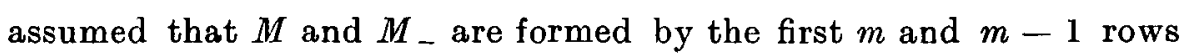
and columns of $A$ respectively, which can always be attained by permuting the rows and columns of $A$ in the same manner. In Part III the following generalisation of 2.1 will be obtained:

2.2. If all $\left|M_{-}^{+}\right| \neq 0$ while all $\left|M_{-}^{++}\right|$and $\left|M^{++}\right|$vanish, then $r=m$. 2.1 can, however, also be obtained by means of $\S 7$.

For special matrices $A$ the conditions of 2.2 can be replaced by weaker ones ${ }^{2}$ :

2.3. If $|M| \neq 0$ while all $\left|M^{+}\right|$and $\left|M^{++}\right|$vanish, then the rank of the symmetrical (or antisymmetrical) matrix $A$ is $m$.

At the same time reasons will appear for the failure of a similar statement recently given by W. H. Metzler ${ }^{3}$ for general matrices.

\section{Minors of an arbitrary order.}

§3. Before proving 1.2 and 1.3 we will establish the two following lemmas ${ }^{4}$ :

3.1. If $|A|$ and all the coaxial minors of $A$ vanish, then all the $n$ ! terms of $|A|=\Sigma \pm a_{11} a_{22} \ldots a_{n n}$ vanish.

1 Gött. Nachrichten (1899), 272-281.

2 Kantor, loc. cit., Frobenius, Joumal für Math., 82 (1877), 230-315, and Sitzb. derPreuss. Akad. Wiss. (1894), 241-256.

3 Amer. Math. Monthly, XLI (1934), 607.608.

4 These are, for $n>3$, contained in 1.3 . 
3.2. If $; A \neq 0$, while all the coaxial minors of $A$ vanish, then $A, b y$ permuting its rows and columns in the same manner, can be brought into the form 1.32 .

The lemma 3.1 is trivial for $n=1$ and $n=2$; we may, therefore, assume it valid for all orders less than $n$.

Then every term $\mu$ of a coaxial minor of $A$ vanishes, and thus also every term of $A \mid$ vanishes in which $\mu$ appears as a factor, i.e. which contains a cycle of less than $n$ indices. If, now, 3.1 were not true, one of the remaining terms, say $a=a_{12} a_{23} \ldots a_{n 1}$, would be different from zero. But then, every $a_{i k}$ not contained in $a$ must be zero; e.g. $a_{52}$, because of $\mu=a_{23} a_{34} a_{45} a_{52}=0, a_{23} a_{34} a_{45} \neq 0$. Therefore we have $\mid A j= \pm \alpha \neq 0$, while it was assumed that $|A|=0$.

Passing to $3.2,3.1$ applies to every coaxial minor of $A$, so again every term of $|A|$ vanishes which contains a cycle of less than $n$ indices. As $|A| \neq 0$, one of the remaining terms, say the above $a$, must be different from zero. As before every $a_{i k}$ not contained in $a$ is seen to ranish, so $A$ has the form 1.32 .

\$4. In order to prove 1.2 , let us first remark that, under the suppositions there made, every non-vanishing coaxial minor $|M|$ of order $n-m$ of $A$ contains by 3.2 exactly $n-m$ non-vanishing elements. To these elements there corresponds a cycle $\mu$ of $n-m$ indices which may be called a non-ranishing cycle, and no shorter cycle of the same kind exists. Incidentally we see that the number $m$ belonging to such a given matrix $A$ depends only upon the position of the zero elements of $A$.

We now remark that in our matrix $A$ any three cycles $\mu_{0}, \mu_{1}$, $\mu_{2}$ of $n-m$ indices must have at least one common index. For let $\nu_{0}$ denote the set composed of those indices $1,2, \ldots, n$ which are not contained in $\mu_{0}$, and define $\nu_{1}, \nu_{2}$ similarly. As $\nu_{0}, \nu_{1}, \nu_{2}$ contain each $m$ indices and together at most $3 m<n$ indices, there must exist indices belonging to neither of them and therefore common to $\mu_{0}, \mu_{1}, \mu_{2}$.

$\S 5$. From these cycles $\mu_{0}, \mu_{1}, \mu_{2}, \ldots$ a rectangular matrix $M$ can be constructed as follows. In the first line put the indices belonging to $\mu_{0}$, beginning with an arbitrary index and following each other as in $\mu_{0}$. The next Yine is similarly formed of the indices belonging to $\mu_{1}$, beginning, however, in a way to make at least one common index 
$i$ of $\mu_{0}$ and $\mu_{1}$ (which by $\S 4$ exists) stand in both in the same column. If another common index $k$ would be found in different columns, as in

$$
\begin{aligned}
& \mu_{0}=\left(\begin{array}{lllll}
\alpha & i \beta & k & \gamma
\end{array}\right) \text {. } \\
& \mu_{1}=\left(\begin{array}{ll}
\delta i & \epsilon k \zeta
\end{array}\right),
\end{aligned}
$$

where $a, \beta, \ldots$ denote groups of indices, a shorter non-vanishing cycle $\left(\delta i \beta k \zeta\right.$ ) could be constructed by combining indices of $\mu_{0}$ and $\mu_{1}$. So if one common index of two cycles stands in both in the same column, the same is true for all common indices.

Now put the indices of $\mu_{2}$ in the third line of $M$, making, as before, its common indices with $\mu_{0}$ stand in both in the same column, and similarly for the other lines. Then every two lines of $M$, say $\mu_{1}$ and $\mu_{2}$, have eo ipso their common indices standing in the same column, because this is true for their common index with $\mu_{0}$ which exists by $\S 4$.

$\S 6$. The formation of the columns of $M$ is thus seen to be independent of the choice of $\mu_{0}$, and no two of these columns have an index in common.

Let the number of different indices contained in the $n-m$ columns of $M$ be $c_{1}, c_{2}, \ldots, c_{n-m}$; then the number of different non-vanishing cycles of $n-m$ indices, i.e. of lines of $M$, is clearly at most $c=\prod_{\lambda=1}^{n-m} c_{\lambda}$. The sum of a possible set of $c_{\lambda}$ 's must be less than or equal to $n$; at least $n-2 m$ of the $c_{\lambda}$ 's, therefore, are unity. Modifying a given set with $c_{\kappa}>2, c_{\lambda}=1$ by putting $c^{\prime}{ }_{k}=c_{\kappa}-1, c^{\prime}{ }_{\lambda}=2$, we get a $c^{\prime}>c$; the greatest value of $c$ will thus be obtained if all $c_{\lambda}$ 's are unity or two, the number of the latter being at most $m$.

As matrices $A$ can be found without difficulty such that the number of $c_{\lambda}$ 's equal to two is exactly $m$ and the number of lines of $M$ exactly $c$, the upper bound required is $c=2^{m}$, as stated in 1.2.

As a concrete example with $n=7, m=2$, put

$$
A=\left[\begin{array}{lllllll}
0 & . & 0 & 0 & 0 & . & 0 \\
0 & 0 & . & 0 & 0 & a & 0 \\
0 & 0 & 0 & . & 0 & 0 & . \\
0 & 0 & 0 & 0 & . & 0 & b \\
. & 0 & 0 & 0 & 0 & 0 & 0 \\
0 & c & . & 0 & 0 & 0 & 0 \\
0 & 0 & 0 & d & . & 0 & 0
\end{array}\right], a c=b d=0
$$


where the points denote arbitrary non-vanishing numbers; for this $A$, we obtain

$$
M=\left(\begin{array}{lllll}
1 & 2 & 3 & 4 & 5 \\
1 & 2 & 3 & 7 & 5 \\
1 & 6 & 3 & 4 & 5 \\
1 & 6 & 3 & 7 & 5
\end{array}\right)
$$

$c_{1}=c_{3}=c_{5}=1, c_{2}=c_{4}=2, c=4$.

$\S 7$. Concerning the related question about the matrices $A$ with $|\boldsymbol{A}|=0$ whose coaxial minors of every order greater than $n-m$ vanish, it may be mentioned that in the simplest case $m=2$ these matrices consist (i) of the matrices of rank less than $n-1$; (ii) of those matrices whose diagonal elements can be divided in two classes I and II such that neither the rows containing I nor the columns containing II are linearly independent.

§. Let us proceed to the enumeration given by 1.3. By 1.2 for $m=1$ there are at most two non-vanishing coaxial first minors of $A$. If there are exactly two, the corresponding cycles $\mu$ of $n-1$ indices must have their common indices in the same order $(\$ 5), e . g$. $(1245 \ldots n)$ and $(1345 \ldots n)$; so, remembering that $a_{23} a_{32}=0$, we obtain the last two cases of 1.3 .

Further, if the one existing $\mu$ is $(23 \ldots n), a_{1 k} \neq 0$ and $a_{i 1} \neq 0$ would belong to a cycle of less than $n$ non-vanishing elements, so then either all $a_{1 k}$ or all $a_{i 1}$ must vanish; this gives 1.34 and 1.33 respectively.

Finally, if all coaxial minors of $A$ including the first minors vanish, $|A| \neq 0$ implies 1.32 as stated in 3.2 : and if $|A|=0$, the rearrangement of its indices $i=1,2, \ldots, n$ which brings $A$ in the form 1.31 can be made as follows.

§9. If, for a given pair of indices $i, k$, there exists a product of the form $a=a_{i i_{1}} a_{i_{1} i_{2}} \ldots a_{i_{\lambda} k} \neq 0$ (including the case of a product consisting of one element only, i.e. $a=a_{i k} \neq 0$ ), we say that $i$ is "prior" to $k$; then in virtue of 3.1 cycles of indices cannot appear in $a$, and all the indices $i, i_{1}, \ldots, i_{\lambda}, k$ must be different from one another. Therefore we cannot have $i$ prior to itself. Moreover, if $i$ is prior to $k$ and $k$ is prior to $l$, then $i$ is prior to $l$. Hence $i$ and $k$ cannot both be prior to the other. 
But there may be pairs of indices between which no relation of priority holds. There may even exist indices which do not appear in any such relation; let their set be $J_{0}$ (which may be void). Let $J_{1}$ be the set of those $i$ 's which have no prior $k$, generally $J_{\lambda}$ be the set of those $i$ 's which are not contained in $J_{0}, J_{1}, \ldots, J_{\lambda-1}$ and which have no prior $k$ except in these sets; thus all the indices $i$ belong to the finite number of non-void $J$ 's. We then fix an arbitrary order between the indices belonging to the same $J$, and place the indices belonging to $J_{\lambda}$ after those belonging to $J_{\lambda-1}$.

In the new order, $a_{i k} \neq 0$ implies $i$ is prior to $k$; if, therefore, the rows and columns of $A$ are ordered accordingly, $A$ has the form 1.31 ; this completes the proof of 1.3 .

\section{Minors of two consecutive orders.}

$\S 10$. Let us first establish a decomposition for an arbitrary square matrix $A$ which has a non-vanishing coaxial minor $M$. $M$ may be assumed to be formed by the first $m$ rows and columns of $A$. The remaining rows and columns of $A$ intersect in another coaxial minor $M_{3}$, so $A$ may be written

10.1 .

$$
A=\left(\begin{array}{cc}
M & M_{1} \\
M_{2} & M_{3}
\end{array}\right)=\left(a_{i k}\right), \quad i, k=1,2, \ldots, n,
$$

thus defining $M_{1}$ and $M_{2}$ as rectangular matrices.

Then, as $M \neq 0$, using the symbols $X Y$ and $X^{-1}$ for the matrix product and inverse matrix, we can put

$\begin{array}{lll}M^{-1} M_{1}=N=\left(n_{i k}\right), & i=1,2, \ldots, m, & k=1,2, \ldots, n-m ; \\ M_{2} M^{-1}=P=\left(p_{i k}\right), & i=1,2, \ldots, n-m, & k=1,2, \ldots, m \\ M_{3}-P M N=Q=\left(q_{i k}\right), & i=1,2, \ldots, n-m, & k=1,2, \ldots, n-m ;\end{array}$

so that 10.1 becomes

$$
A=\left(\begin{array}{cc}
M & M N \\
P M & P M N+Q
\end{array}\right) .
$$

Conversely, the matrices $M, N, P, Q$, with the indicated numbers of rows and columns, may be prescribed arbitrarily and independently of one another, and $A$ is then uniquely given by 10.2 .

Subtracting appropriate multiples of the first $m$ columns of $A$ from the other columns we obtain

$$
|A|=\left|\begin{array}{cc}
M & 0 \\
P M & Q
\end{array}\right|=|M||Q|
$$

by use of Laplace's theorem. An analogous relation holds for every 
minor of $A$ containing $M$. Therefore, as $M \mid \neq 0$, we can state that 10.3. Any minor of $A$ containing $M$ as a minor vanishes if, and only if, the corresponding minor of $Q$ vanishes.

Thus $r=m$ implies $Q \equiv 0$, i.e. all $q_{i k}=0$; conversely, from $Q \equiv 0$ it follows by 10.2 that the last $n-m$ columns of $A$ are linear combinations of the first $m$ columns. As $|M| \neq 0$, the rank $r$ cannot be less than $m$. So we have' ${ }^{1}$ :

10.4. If $|M| \neq 0, r=m$ holds if, and only if, $Q \equiv 0$.

\$11. It is equally easy to express the vanishing of all $M^{+}$and $M^{++}$ as a property of $Q$. We deduce first from 10.3 that $q_{i i}=0$ for all $i$, and then that

11.1. If $\mid M \neq 0$, all $\mid M^{+}:$and,$M^{++} \mid$vanish if, and only if, all $q_{i k} q_{k i}$ vanish.

The suppostions of 11.1 are of course contained in those of 10.4. Let $C$ be a condition which, taken in conjunction with the former, will imply the latter. We may remark that even the vanishing of all coaxial minors of $A$ containing $M$ as a minor is not a condition $C$ as it only ensures that $Q$ is of the forms 1.31 or 1.32 (after a certain permutation), so that $r$ may be any number between $m$ and $n$ inclusive.

W. H. Metzler's statement ${ }^{2}$ amounts to saying that, $M_{-}$being given, "all $M_{-}+$but one do not vanish" is such a condition $C$. This, however, can be replaced by " $n_{m i} \neq 0, p_{i m} \neq 0$ for all $i$ 's but one," conditions which do not affect $Q$ and are thus irrelevant for the truth of $r=m$. Counter examples are indeed easily found, e.g. for $m=2$,

$$
A_{i}=\mid \begin{array}{lllll}
1 & 0 & 0 & 0 & 0 \\
0 & 1 & 0 & 1 & 1 \\
0 & 0 & 0 & 1 & 0 \\
0 & 1 & 0 & 1 & 2 \\
0 & 1 & 1 & 1 & 1
\end{array} .=1 .
$$

\$12. To conclude that $q_{i k}=0$ from $q_{i k} q_{k i}=0$ it is however sufficient to assume $q_{i k}=q_{k i}$ or $q_{i k}=-q_{k i}$. But $q_{i k}= \pm q_{k i}, n_{i k}=p_{k i}$ is a consequence of $a_{i k}= \pm a_{k i}$ for all $i, k$. Hence this last assumption is a condition $C$, whereby 2.3 is established.

${ }^{1}$ Kronecker, Journal für Math., 72 (1870), 152-175.

2 loc cit., p. 
Another condition $C$, of a different kind from the previous one and similar to Metzler's condition, is given by 2.2. We verify it by remarking that a $\left|M_{-}^{++}\right|$which is not a $M+$ can be written:

$$
\left|M_{-}^{++}\right|=\left|\begin{array}{ccc} 
& a_{1 m} n_{m i} & a_{1 m} n_{m k} \\
M_{-} & \ldots & \ldots \ldots \\
& a_{m-1 m} n_{m i} & a_{m-1 m} n_{m k} \\
p_{i m} a_{m 1} \ldots p_{i m} a_{m m-1} & p_{i m} a_{m m} n_{m i} & p_{i m} a_{m m} n_{m k}+q_{i k} \\
p_{k m} a_{m 1} \ldots p_{k m} a_{m m-1} & p_{k m} a_{m m} n_{m i}+q_{k i} & p_{k m} a_{m m} n_{m k}
\end{array}\right|
$$

As the minor $\left|M_{i}\right|$, formed by the first $m$ rows and columns of this $\left|M_{-}{ }^{++}\right|$, is a $\left|M_{-}+\right|$, its factors $n_{m i}$ and $p_{i m}$ must be different from zero; similarly $n_{m k} \neq 0, p_{k m} \neq 0$. If now $q_{i k}=0$, say, $q_{k i} \equiv 0$, we subtract the last row but one of this $\left|M_{-}{ }^{++}\right|$, multiplied by $p_{k m} / p_{i m}$, from the last row, getting $\left|M_{-}{ }^{++}\right|=\left(-q_{k i} n_{m k} / n_{m i}\right) i M_{i} \mid$. Hence $q_{k i}=0, Q \equiv 0$, and by 10.4 we obtain 2.2 . 